\title{
EVIDENCE FOR THE GROMOV-WITTEN/DONALDSON-THOMAS CORRESPONDENCE
}

\author{
Amin Gholampour and Yinan Song
}

\begin{abstract}
We study the equivariant Gromov-Witten and Donaldson-Thomas theories of $\mathbf{P}^{2}$-bundles over curves. We show the equivariant GW/DT correspondence holds to first order for certain curve classes.
\end{abstract}

\section{Introduction}

Both Gromov-Witten and Donaldson-Thomas theory arise from the enumerative geometry of threefolds. In [4], [5], and [1], correspondences between these two theories were conjectured in various settings. In this paper, we provide evidence for the equivariant conjecture in the case where the threefold is a $\mathbf{P}^{2}$-bundle over a curve. The equivariant Gromov-Witten theory of $\mathbf{P}^{2}$-bundle over curves is solved in [2] for certain curve classes, but we can only compute the Donaldson-Thomas invariants up to first order. Still, our computation is the first compact non-toric example where the correspondence has been checked.

Let $C$ be a smooth and connected curve of genus $g$. Let $L_{1}$ and $L_{2}$ be line bundles of degrees $k_{1}$ and $k_{2}$, and let $X$ be the total space of the $\mathbf{P}^{2}$-bundle $\mathbf{P}\left(\mathcal{O} \oplus L_{1} \oplus L_{2}\right)$ over $C$. The three dimensional torus, $\left(\mathbf{C}^{*}\right)^{3}$ acts diagonally on the fibers of $X$. We denote by $t_{0}, t_{1}$ and $t_{2}$, the equivariant parameters of this action along the first, second and third summand, respectively. Let $\beta$ be a curve class in $X$.

Following [2], we define the partition function for the degree $\beta$ equivariant GromovWitten invariants of $X$ as:

$$
Z_{\beta}^{G W}\left(g \mid k_{1}, k_{2}\right)=\sum_{h=0}^{\infty} u^{2 h-2-K_{X} \cdot \beta} \int_{\left[\bar{M}_{h}^{\bullet}(X, \beta)\right]^{v i r}} 1,
$$

where $\bar{M}_{h}^{\bullet}(X, \beta)$ is the moduli space of the genus $h$, degree $\beta$, possibly disconnected stable maps to $X$, and $K_{X}$ is the canonical class of $X$. The integration in the above formula denotes equivariant pushforward to a point, so it can be non-zero for negative virtual dimension. The equivariant Gromov-Witten partition function will be an element in

$$
\mathbf{Q}((u))\left[t_{0}, t_{1}, t_{2}\right] .
$$

Remark 1. It is worth emphasizing that when the virtual dimension of the moduli space is positive then the partition function is zero (See [2], Section 1).

Received by the editors October 21, 2005. 
As in [1], we define the the partition function for the class $\beta$ equivariant DonaldsonThomas invariants of $X$ as

$$
Z_{\beta}^{D T}\left(g \mid k_{1}, k_{2}\right)=\sum_{n \in \mathbb{Z}} q^{n} \int_{\left[I_{n}(X, \beta)\right]^{v i r}} 1,
$$

where $I_{n}(X, \beta)$ is the moduli space of ideal sheaves parameterizing subschemes $Y$ in $X$, whose maximal one dimensional component is in the class $\beta$ and $\chi\left(O_{Y}\right)=n$. Again, the integral denotes equivariant pushforward to a point. The Donaldson-Thomas partition function is an element in

$$
\mathbf{Z}((u))\left[t_{0}, t_{1}, t_{2}\right] .
$$

It takes coefficients in $\mathbf{Z}((u))$, rather than $\mathbf{Q}((u))$ because $I_{n}(X, \beta)$ is a scheme, so the virtual class is defined over $\mathbf{Z}$.

We define the reduced Donaldson-Thomas partition function by

$$
Z_{\beta}^{D T}\left(g \mid k_{1}, k_{2}\right)^{\prime}=M(-q)^{-\int_{X} c_{3}\left(T_{X} \otimes K_{X}\right)} \cdot Z_{\beta}^{D T}\left(g \mid k_{1}, k_{2}\right),
$$

where

$$
M(q)=\prod_{n \geq 1} \frac{1}{\left(1-q^{n}\right)^{n}}
$$

is the McMahon function, and $T_{X}$ is the tangent bundle of $X$ (See Definition 9.2, and Conjecture 1, in[1]).

In this notation, GW/DT correspondence conjecture in [1] reads as follows:

GW/DT Conjecture. After the change of variable, $e^{i u}=-q$, we have

$$
(-i)^{-K_{X} \cdot \beta} Z_{\beta}^{G W}\left(g \mid k_{1}, k_{2}\right)=(-q)^{\frac{1}{2} K_{X} \cdot \beta} Z_{\beta}^{D T}\left(g \mid k_{1}, k_{2}\right)^{\prime} .
$$

We define $\beta_{0}$ to be the curve class of the section given by the locus $(1: 0: 0)$, and $f$ to be the line class of a fibre in $X$. Then we prove the following result:

Theorem 2. $G W / D T$ Conjecture holds for the leading term in $q$, when $\beta=\beta_{0}$ and $\beta=\beta_{0}+f$, and $k_{1}, k_{2} \leq 0$.

The case $\beta=\beta_{0}$ is straightforward. We will prove the theorem for $\beta=\beta_{0}+f$, and when $k_{1}, k_{2}<-1$, where we can express our results more uniformly. The case, where one or both of $k_{1}$ and $k_{2}$ are equal to -1 or 0 , is similar. In Section 2 , we will compute the left hand side of (1) (Proposition 3). In section 3, we will compute the first term of the series in the right hand side of (1) (Proposition 4). Theorem 2 will follow after changing the variable, $e^{i u}=-q$, and comparing the coefficients of $q$ with the lowest power in the both sides of (1).

\section{The Gromov-Witten Theory of $X$}

Let $X$ and $\beta=\beta_{0}+f$ be as above. We can express the result of this section as follows: 
Proposition 3. Let $k_{1}, k_{2}<-1$, then the partition function for the degree $\beta=\beta_{0}+f$ equivariant Gromov-Witten invariants of $X$ is given by

$$
\begin{aligned}
& Z_{\beta}\left(g \mid k_{1}, k_{2}\right)=\left(t_{0}-t_{1}\right)^{g-k_{1}-3}\left(t_{0}-t_{2}\right)^{g-k_{2}-3} \\
& \left.\quad \cdot\left(\left(4 g-4-k_{1}-k_{2}\right) t_{0}-\left(2 g-2-k_{2}\right) t_{1}-\left(2 g-2-k_{1}\right) t_{2}\right)\right)\left(2 \sin \frac{u}{2}\right)^{k_{1}+k_{2}+3} .
\end{aligned}
$$

In [2], the section class equivariant Gromov-Witten theory of $X$ was completely determined. The following matrices were defined in [2]:

$$
\begin{gathered}
A=\left[\begin{array}{ccc}
\left(t_{0}-t_{1}\right)\left(t_{0}-t_{2}\right) & 0 & 0 \\
0 & \left(t_{1}-t_{0}\right)\left(t_{1}-t_{2}\right) & 0 \\
0 & 0 & \left(t_{2}-t_{0}\right)\left(t_{2}-t_{1}\right)
\end{array}\right], \\
B=\left[\begin{array}{ccc}
\frac{2\left(2 t_{0}-t_{1}-t_{2}\right)}{\left(t_{0}-t_{1}\right)\left(t_{0}-t_{2}\right)} & \frac{t_{0}+t_{1}-2 t_{2}}{\left(t_{0}-t_{1}\right)\left(t_{0}-t_{2}\right)} & \frac{t_{0}+t_{2}-2 t_{1}}{\left(t_{0}-t_{1}\right)\left(t_{0}-t_{2}\right)} \\
\frac{t_{0}+t_{1}-2 t_{2}}{\left(t_{1}-t_{0}\right)\left(t_{1}-t_{2}\right)} & \frac{2\left(2 t_{1}-t_{0}-t_{2}\right)}{\left(t_{1}-t_{0}\right)\left(t_{1}-t_{2}\right)} & \frac{t_{1}+t_{2}-20_{0}}{\left(t_{1}-t_{0}\right)\left(t_{1}-t_{2}\right)} \\
\frac{t_{0}+t_{2}-2 t_{1}}{\left(t_{2}-t_{0}\right)\left(t_{2}-t_{1}\right)} & \frac{t_{1}+t_{2}-2 t_{0}}{\left(t_{2}-t_{0}\right)\left(t_{2}-t_{1}\right)} & \frac{2\left(22 t_{2}+t_{0}-t_{1}\right)}{\left(t_{2}-t_{0}\right)\left(t_{2}-t_{1}\right)}
\end{array}\right] \phi^{3} . \\
M_{1}=\left[\begin{array}{ccccc}
t_{0}-t_{1} & 0 & 0 \\
0 & 0 & 0 \\
0 & 0 & t_{2}-t_{1}
\end{array}\right] \phi^{-1}, \quad M_{2}=\left[\begin{array}{ccc}
t_{0}-t_{2} & 0 & 0 \\
0 & t_{1}-t_{2} & 0 \\
0 & 0 & 0
\end{array}\right] \phi^{-1}, \\
N=\left[\begin{array}{cccc}
\frac{1}{\left(t_{0}-t_{1}\right)\left(t_{0}-t_{2}\right)} \\
\frac{1}{\left(t_{0}-t_{1}\right)\left(t_{0}-t_{2}\right)} & \frac{1}{\left(t_{0}-t_{1}\right)\left(t_{0}-t_{2}\right)\left(t_{1}-t_{2}\right)} \\
\frac{\left(t_{1}-t_{0}\right)\left(t_{1}-t_{2}\right)}{\left(t_{1}-t_{0}\right)\left(t_{1}-t_{2}\right)} & \frac{1}{\left(t_{1}-t_{0}\right.} \\
\frac{1}{\left(t_{2}-t_{0}\right)\left(t_{2}-t_{1}\right)} & \frac{1}{\left(t_{2}-t_{0}\right)\left(t_{2}-t_{1}\right)} & \frac{1}{\left(t_{2}-t_{0}\right)\left(t_{2}-t_{1}\right)}
\end{array}\right] \phi^{2},
\end{gathered}
$$

where $\phi=2 \sin \frac{u}{2}$.

In [2], $G=A+B$ was called the genus adding operator, and $U_{1}^{-1}=M_{1}+N$ and $U_{2}^{-1}=M_{2}+N$ were called the first and the second level annihilation operator, respectively.

Notation. For two matrices $U$ and $V$, by $\left(U^{a}, V^{b}\right)$ for $a, b \in \mathbb{Z}^{+}$, we mean the sum of the all products that we can write containing $a$ copies of $U$ and $b$ copies of $V$. For example

$$
\left(U^{2}, V\right)=U^{2} V+U V U+V U^{2} .
$$

Now by Theorem 1.7 in [2], for $k_{1}, k_{2} \leq 0$, and $r \geq 0$, we have

$$
Z_{\beta_{0}+r f}\left(g \mid k_{1}, k_{2}\right)=\sum \operatorname{tr}\left(\left(A^{a}, B^{b}\right)\left(M_{1}^{m_{1}}, N^{n_{1}}\right)\left(M_{2}^{m_{2}}, N^{n_{2}}\right)\right),
$$

where the sum is over all nonnegative integers $a, b, m_{1}, m_{2}, n_{1}$, and $n_{2}$ such that

$$
a+b=g-1, \quad m_{1}+n_{1}=-k_{1}, \quad m_{2}+n_{2}=-k_{2}, \quad b+n_{1}+n_{2}=r .
$$

Proof of Proposition 3: By the discussion above, for $\beta=\beta_{0}+f$, we have

$$
\begin{aligned}
& Z_{\beta}\left(g \mid k_{1}, k_{2}\right)= \\
& \operatorname{tr}\left(\left(A^{g-2}, B\right) M_{1}^{-k_{1}} M_{2}^{-k_{2}}+A^{g-1}\left(M_{1}^{-k_{1}-1}, N\right) M_{2}^{-k_{2}}+A^{g-1} M_{1}^{-k_{1}}\left(M_{2}^{-k_{2}-1}, N\right)\right) .
\end{aligned}
$$


Now since $\operatorname{tr}(U V)=\operatorname{tr}(V U)$ for any two matrices, and also since $A, M_{1}$, and $M_{2}$ mutually commute, this is equal to

$$
\begin{aligned}
& (g-1) \operatorname{tr}\left(A^{g-2} M_{1}^{-k_{1}} M_{2}^{-k_{2}} B\right)-k_{1} \operatorname{tr}\left(A^{g-1} M_{1}^{-k_{1}-1} M_{2}^{-k_{2}} N\right) \\
& -k_{2} \operatorname{tr}\left(A^{g-1} M_{1}^{-k_{1}} M_{2}^{-k_{2}-1} N\right) .
\end{aligned}
$$

From this, Proposition 3 easily follows.

\section{The Donaldson-Thomas Theory of $X$}

Let $X$ and $I_{n}(X, \beta)$ be as in Section 1 , and let $\pi$ be the projection from $X$ to $C$. Unlike the computation in Gromov-Witten theory, we can only compute the first term of the partition function in Donaldson-Thomas theory in the right hand side of (1). The power of $q$ of the first term is the holomorphic Euler characteristic of the structure sheaf of a purely 1-dimensional subscheme, $Y$, in the class $\beta$, which is equal to $\chi\left(\mathcal{O}_{Y}\right)=1-g$. From now on we fix

$$
n=1-g .
$$

By definition, the coefficient of the first term of the right hand side of (1) is

$$
N_{n}^{D T}(X, \beta)=\int_{\left[I_{n}(X, \beta)\right]^{v i r}} 1=\int_{\left[I_{n}(X, \beta)^{T}\right]^{v i r}} \frac{1}{e\left(\mathrm{Norm}^{\text {vir }}\right)},
$$

where $I_{n}(X, \beta)^{T}$ is the fixed loci of the induced torus action on $I_{n}(X, \beta)$, and Norm ${ }^{\text {vir }}$ is its virtual normal bundle, and $e$ denotes equivariant Euler class, see [1] for a detailed discussion. Now we express the result of this section:

Proposition 4. Let $X, n$, and $k_{1}, k_{2}<-1$ be as above. Then the equivariant Donaldson-Thomas invariant of class $\beta=\beta_{0}+f$ and holomorphic Euler characteristic $n$ is given by:

$$
\begin{aligned}
N_{n}^{D T}(X, \beta)= & \left(t_{0}-t_{1}\right)^{g-k_{1}-3}\left(t_{0}-t_{2}\right)^{g-k_{2}-3} \\
& \left.\cdot\left(\left(4 g-4-k_{1}-k_{2}\right) t_{0}-\left(2 g-2-k_{2}\right) t_{1}-\left(2 g-2-k_{1}\right) t_{2}\right)\right) .
\end{aligned}
$$

Before giving the proof, we first describe the fixed loci explicitly:

Lemma 5. $I_{n}(X, \beta)^{T}$ is isomorphic to the union of two disjoint copies of $C$, and moreover the universal subscheme in $I_{n}(X, \beta)^{T} \times X$ is a complete intersection.

Proof: Let $M=C_{1} \amalg C_{2}$ where $C_{1}$ and $C_{2}$ are two copies of $C$. We will consider $M$ as $\operatorname{Hilb}^{1}(M)$ and show that $M$ is isomorphic to $I_{n}(X, \beta)^{T}$ by showing that they represent isomorphic functors of points. In other words, we will show how to construct a family of one-point in $\mathrm{M}$ from a family of fixed subschemes and vice versa. First we construct an $M$-family of fixed subschemes:

$$
\mathcal{Y} \subset M \times X \stackrel{p r_{1}}{\rightarrow} M,
$$

where $p r_{1}$ and $p r_{2}$ denote projections from $M \times X$ onto $M$ and $X$ respectively. We define $\Delta$ to be the diagonal divisor in $C \times C$. We use $\Delta_{1}$ and $\Delta_{2}$ to distinguish between the two diagonals in $M \times C$. We use id $\times \pi$ denote from the map from $M \times X$ to $M \times C$. 
The projective bundle $X$ can be constructed in three ways, as $\mathbf{P}\left(\mathcal{O} \oplus L_{1} \oplus L_{2}\right)$, and $\mathbf{P}\left(L_{1}^{*} \oplus \mathcal{O} \oplus L_{2} L_{1}^{*}\right)$, and $\mathbf{P}\left(L_{2}^{*} \oplus L_{1} L_{2}^{*} \oplus \mathcal{O}\right)$. The tautological bundles change depending on the construction. We use the following notation for the hyperplane divisors:

$$
\begin{gathered}
H_{0}=\mathbf{P}\left(L_{1} \oplus L_{2}\right) \subset X=\mathbf{P}\left(\mathcal{O} \oplus L_{1} \oplus L_{2}\right) \\
H_{1}=\mathbf{P}\left(L_{1}^{*} \oplus L_{2} L_{1}^{*}\right) \subset X=\mathbf{P}\left(L_{1}^{*} \oplus \mathcal{O} \oplus L_{2} L_{1}^{*}\right) \\
H_{2}=\mathbf{P}\left(L_{2}^{*} \oplus L_{1} L_{2}^{*}\right) \subset X=\mathbf{P}\left(L_{2}^{*} \oplus L_{1} L_{2}^{*} \oplus \mathcal{O}\right)
\end{gathered}
$$

The corresponding line bundles are $\mathcal{O}_{X}(1), \pi^{*} L_{1} \otimes \mathcal{O}_{X}(1)$ and $\pi^{*} L_{2} \otimes \mathcal{O}_{X}(1)$. Finally, we let $D_{1}=p r_{2}^{*} H_{1}$ and $D_{2}=p r_{2}^{*} H_{2} \cup(\mathrm{id} \times \pi)^{*} \Delta_{1}$ and $D_{1}^{\prime}=p r_{2}^{*} H_{2}$ and $D_{2}^{\prime}=p r_{2}^{*} H_{1} \cup(\mathrm{id} \times \pi)^{*} \Delta_{2}$.

The family $\mathcal{Y}$ is a disjoint union of two components $\mathcal{Y}_{1}$ and $\mathcal{Y}_{2}$. One component is the intersection of $D_{1}$ and $D_{2}$ and the other is the intersection of $D_{1}^{\prime}$ and $D_{2}^{\prime}$. This family is fixed under the $T$-action.

This family $\mathcal{Y}$ is constructed out of the universal family of $\operatorname{Hilb}^{1}(M)$. Therefore, given a $B$-family of one-point in $M$, which is equivalent to a map from $B$ to $M$, we can pull back the family $\mathcal{Y}$ to obtain a family of $T$-fixed subschemes over $B$.

Conversely, let's consider a $B$-family $\mathcal{Z}$ of fixed subschemes. We can intersect them with the hyperplane divisor at the infinity, $H_{0}$. Since the subschemes are of the class $\beta_{0}+f$, each fixed scheme intersects $H_{0}$ at a point. This point is a $T$-fixed point, so it is a point on either the locus of $(0: 1: 0)$ in $X=\mathbf{P}\left(L_{1}^{*} \oplus \mathcal{O} \oplus L_{2} L_{1}^{*}\right)$ or the locus of $(0: 0: 1)$ in $X=\mathbf{P}\left(L_{2}^{*} \oplus L_{1} L_{2}^{*} \oplus \mathcal{O}\right)$. The union of these two loci is isomorphic to $M$. Therefore, we get a $B$-family of one-points in $M$. It is easy to see that it is an isomorphism of functors, so we get an isomorphism of the moduli spaces.

Proof of Proposition 4: Consider the first component constructed in the previous lemma. For convenience, we will suppress the subscript in $C_{1}$ and $\mathcal{Y}_{1}$ from now on. Recall that $\pi$ and id $\times \pi$ are the projections from $\mathrm{X}$ and $C \times X$ to $\mathrm{C}$ and $C \times C$ respectively, that $p r_{1}$ and $p r_{2}$ are projections from $C \times X$ onto $C$ and $X$ respectively, and that $p_{1}$ and $p_{2}$ are projections from $C \times C$ onto the first and the second factor respectively. Recall that $\mathcal{Y}$ is a zero section of rank two vector bundle on $C \times X$, which is the direct sum of the following two line bundles:

$$
\begin{aligned}
& \mathcal{O}_{C \times X}\left(D_{1}\right)=p r_{2}^{*}\left(\pi^{*} L_{1} \otimes \mathcal{O}_{X}(1)\right) \\
& \mathcal{O}_{C \times X}\left(D_{2}\right)=p r_{2}^{*}\left(\pi^{*} L_{2} \otimes \mathcal{O}_{X}(1)\right) \otimes(\mathrm{id} \times \pi)^{*} \mathcal{O}_{C \times C}(\Delta) .
\end{aligned}
$$

The K-theory class of the perfect obstruction theory on $C$, considered as a connected component of $I_{n}(X, \beta)^{T}$, is given by

$$
\mathbf{R}^{\bullet} \operatorname{pr}_{1, *}\left(\mathcal{H o m}(\mathcal{I}, \mathcal{I})-\mathcal{O}_{C \times X}\right),
$$

where $\mathcal{I}$ is the universal ideal sheaf (c.f. [4] page 19). According to [3], in terms of equivariant K-theory classes, we can regard

$$
\mathbf{R}^{\bullet} \operatorname{pr}_{1, *}\left(\mathcal{H o m}(\mathcal{I}, \mathcal{I})-\mathcal{O}_{C \times X}\right)=-\operatorname{Def}^{m}-\operatorname{Def}^{f}+\mathrm{Ob}^{m}+\mathrm{Ob}^{f},
$$


where the superscripts, $f$, and $m$, mean the fixed, and the moving parts under the induced action of the torus, respectively. Since the fixed loci is smooth, we will get

$$
N_{n}^{D T}(X, \beta)=\int_{\left[I_{n}(X, \beta)^{T}\right]} \frac{e\left(\mathrm{Ob}^{f}\right) e\left(\mathrm{Ob}^{m}\right)}{e\left(\mathrm{Def}^{m}\right)} .
$$

We will show later that $\mathrm{Ob}^{f}$ is zero, so we can write

$$
N_{n}^{D T}(X, \beta)=\int_{C} e\left(\mathbf{R}^{\bullet} \operatorname{pr}_{1, *}\left(\mathcal{H o m}(\mathcal{I}, \mathcal{I})-\mathcal{O}_{C \times X}\right)\right)^{m} .
$$

Since $\mathcal{Y}$ is the complete intersection of two divisors, $D_{1}$ and $D_{2}$, we have the standard Kozsul resolution of the ideal sheaf of $\mathcal{Y}$ :

$$
0 \rightarrow \mathcal{O}_{C \times X}\left(-D_{1}-D_{2}\right) \rightarrow \mathcal{O}_{C \times X}\left(-D_{1}\right) \oplus \mathcal{O}_{C \times X}\left(-D_{2}\right) \rightarrow \mathcal{I} \rightarrow 0 .
$$

Therefore, the equivariant $\mathrm{K}$-theoretic class of $\mathcal{I}$ is

$$
\mathcal{O}_{C \times X}\left(-D_{1}\right)+\mathcal{O}_{C \times X}\left(-D_{2}\right)-\mathcal{O}_{C \times X}\left(-D_{1}-D_{2}\right) .
$$

Then a formal calculation of equivariant K-theoretic classes gives us

$$
\begin{aligned}
\mathbf{R}^{\bullet} \operatorname{pr}_{1, *}\left(\mathcal{H o m}(\mathcal{I}, \mathcal{I})-\mathcal{O}_{C \times X}\right)= & -\mathbf{R}^{\bullet} \operatorname{pr}_{1, *}\left(\mathcal{O}_{C \times X}\left(D_{1}\right)\right)-\mathbf{R}^{\bullet} \operatorname{pr}_{1, *}\left(\mathcal{O}_{C \times X}\left(D_{2}\right)\right) \\
& -\mathbf{R}^{\bullet} \operatorname{pr}_{1, *}\left(\mathcal{O}_{C \times X}\left(-D_{1}\right)\right)-\mathbf{R}^{\bullet} \operatorname{pr}_{1, *}\left(\mathcal{O}_{C \times X}\left(-D_{2}\right)\right. \\
& +\mathbf{R}^{\bullet} \operatorname{pr}_{1, *}\left(\mathcal{O}_{C \times X}\left(D_{1}-D_{2}\right)+2 \mathbf{R}^{\bullet} p r_{1, *}\left(\mathcal{O}_{C \times X}\right)\right. \\
& +\mathbf{R}^{\bullet} \operatorname{pr}_{1, *}\left(\mathcal{O}_{C \times X}\left(D_{2}-D_{1}\right)\right) .
\end{aligned}
$$

The map, $p r_{1}$, can be factored through

$$
C \times X \stackrel{\text { id } \times \pi}{\rightarrow} C \times C \stackrel{p_{1}}{\rightarrow} C,
$$

where $p_{1}$ is the projection onto the first factor, and therefore

$$
\mathbf{R}^{\bullet} p r_{1, *}=\mathbf{R}^{\bullet} p_{1, *} \circ \mathbf{R}^{\bullet}(\mathrm{id} \times \pi)_{*} .
$$

We will use the following facts in the rest of the proof and we summarize them here in the following lemma:

Lemma 6. With the same notation as in Proposition 4, we have the following identities.

$$
\begin{aligned}
& \mathbf{R}^{\bullet}(\mathrm{id} \times \pi)_{*}\left(\mathcal{O}_{C \times X}\right)=\mathcal{O}_{C \times C}, \\
& \mathbf{R}^{\bullet}(\mathrm{id} \times \pi)_{*}\left(p r_{2}^{*} \mathcal{O}_{X}(1)\right)=p_{2}^{*} L_{0}^{*} \oplus p_{2}^{*} L_{1}^{*} \oplus p_{2}^{*} L_{2}^{*}, \\
& \mathbf{R}^{\bullet}(\mathrm{id} \times \pi)_{*}\left(p r_{2}^{*} \mathcal{O}_{X}(-1)\right)=0, \\
& \mathbf{R}^{\bullet} p_{1, *}\left(p_{2}^{*}\left(L_{0}^{i} L_{1}^{j} L_{2}^{l}\right)\right)=\left(1-g+j k_{1}+l k_{2}\right) \mathbf{C}_{i t_{0}+j t_{1}+l t_{3}}, \\
& \mathbf{R}^{\bullet} p_{1, *}\left(p_{2}^{*}\left(L_{0}^{i} L_{1}^{j} L_{2}^{l}\right) \otimes \mathcal{O}_{C \times C}(-\Delta)=\left(1-g+j k_{1}+l k_{2}\right) \mathbf{C}_{i t_{0}+j t_{1}+l t_{2}}-L_{0}^{i} L_{1}^{j} L_{2}^{l},\right. \\
& \mathbf{R}^{\bullet} p_{1, *}\left(p_{2}^{*}\left(L_{0}^{i} L_{1}^{j} L_{2}^{l}\right) \otimes \mathcal{O}_{C \times C}(\Delta)\right)=\left(1-g+j k_{1}+l k_{2}\right) \mathbf{C}_{i t_{0}+j t_{1}+l t_{2}}+L_{0}^{i} L_{1}^{j} L_{2}^{l} T_{C},
\end{aligned}
$$

where $\mathbf{C}_{s}$ denotes the trivial line bundle on $C$ with weight $-s$, and $L_{0}$ denotes the trivial line bundle on $C \times C$ with weight $-t_{0}$. 
Proof: The first three are elementary. The fourth follows from Riemann-Roch. The last two are proved by applying $\mathbf{R}^{\bullet} p_{1, *}$ to the divisor sequence of $\Delta$ twisted by $p_{2}^{*}\left(L_{0}^{i} L_{1}^{j} L_{2}^{l}\right)$ and $p_{2}^{*}\left(L_{0}^{i} L_{1}^{j} L_{2}^{l}\right) \otimes \mathcal{O}_{C \times C}(\Delta)$ respectively.

Now by the first and fourth equalities in Lemma 6 , we have

$$
\mathbf{R}^{\bullet} \operatorname{pr}_{1, *}\left(\mathcal{O}_{C \times X}\right)=(1-g) \mathbf{C}_{0} .
$$

The second and fourth equalities in Lemma 6 , implies that

$$
\begin{aligned}
\mathbf{R}^{\bullet} p r_{1, *}\left(p r_{2}^{*}\left(\mathcal{O}_{X}(1) \otimes \pi^{*} L_{1}\right)\right)= & \mathbf{R}^{\bullet}\left(p_{1}\right)_{*}\left(\left(p_{2}^{*} L_{0}^{*} \oplus p_{2}^{*} L_{1}^{*} \oplus p_{2}^{*} L_{2}^{*}\right) \otimes p_{2}^{*} L_{1}\right) \\
= & \mathbf{R}^{\bullet}\left(p_{1}\right)_{*}\left(p_{2}^{*}\left(L_{0}^{*} L_{1}\right) \oplus \mathcal{O} \oplus p_{2}^{*}\left(L_{2}^{*} L_{1}\right)\right) \\
= & \left(k_{1}+1-g\right) \mathbf{C}_{t_{1}-t_{0}}+(1-g) \mathbf{C}_{0} \\
& +\left(k_{1}-k_{2}+1-g\right) \mathbf{C}_{t_{1}-t_{2}},
\end{aligned}
$$

and similarly, using the second and the sixth equalities in Lemma 6, we have

$$
\begin{aligned}
\mathbf{R}^{\bullet} p r_{1, *}\left(p r _ { 2 } ^ { * } \left(\mathcal{O}_{X}(1) \otimes\right.\right. & \left.\left.\pi^{*} L_{2}\right) \otimes(\mathrm{id} \times \pi)^{*}\left(\mathcal{O}_{C \times C}(\Delta)\right)\right)= \\
= & \mathbf{R}^{\bullet} p_{1, *}\left(\left(p_{2}^{*} L_{0}^{*} \oplus p_{2}^{*} L_{1}^{*} \oplus p_{2}^{*} L_{2}^{*}\right) \otimes p_{2}^{*} L_{2} \otimes \mathcal{O}_{C \times C}(\Delta)\right) \\
= & \mathbf{R}^{\bullet} p_{1, *}\left(p_{2}^{*}\left(L_{0}^{*} L_{2}\right)(\Delta) \oplus p_{2}^{*}\left(L_{1}^{*} L_{2}\right)(\Delta) \oplus \mathcal{O}_{C \times C}(\Delta)\right) \\
= & \left(1-g+k_{2}\right) \mathbf{C}_{t_{2}-t_{0}}+L_{0}^{*} L_{2} T_{C}+(1-g) \mathbf{C}_{0} \\
& +\left(1-g-k_{1}+k_{2}\right) \mathbf{C}_{-t_{1}+t_{2}}+L_{1}^{*} L_{2} T_{C}+T_{C} .
\end{aligned}
$$

Finally, we can write

$$
\begin{aligned}
\mathbf{R}^{\bullet} \operatorname{pr}_{1, *}\left((\operatorname{id} \times \pi)^{*}\left(p_{2}^{*}\left(L_{1}^{*} L_{2}\right)(\Delta)\right)\right) & =\mathbf{R}^{\bullet}\left(p_{1}\right)_{*}\left(p_{2}^{*}\left(L_{1}^{*} L_{2}\right)(\Delta)\right) \\
& =\left(1-g-k_{1}+k_{2}\right) \mathbf{C}_{-t_{1}+t_{2}}+L_{1}^{*} L_{2} T_{C},
\end{aligned}
$$

and

$$
\begin{aligned}
\mathbf{R}^{\bullet} p r_{1, *}\left((\mathrm{id} \times \pi)^{*}\left(p_{2}^{*}\left(L_{1} L_{2}^{*}\right)(-\Delta)\right)\right) & =\mathbf{R}^{\bullet}\left(p_{1}\right)_{*}\left(p_{2}^{*}\left(L_{1} L_{2}^{*}\right)(-\Delta)\right) \\
& =\left(1-g+k_{1}-k_{2}\right) \mathbf{C}_{t_{1}-t_{2}}-L_{1} L_{2}^{*},
\end{aligned}
$$

where for the first one, we used first and the sixth equalities in Lemma 6 , and for the second one, we used the first and fifth equalities in Lemma 6.

So far, we have computed all the terms in the right hand side of (4), so we can write

$$
\begin{aligned}
e\left(\mathbf{R}^{\bullet} \operatorname{pr}_{1, *}\left(\mathcal{H o m}(\mathcal{I}, \mathcal{I})-\mathcal{O}_{C \times X}\right)\right)^{m}= & \frac{\left(t_{0}-t_{1}\right)^{-k_{1}+g-1}\left(t_{0}-t_{2}\right)^{-k_{2}+g-1}}{\left(\left(t_{0}-t_{2}\right)+\left(k_{2}+2-2 g\right)[p]\right)\left(\left(t_{2}-t_{1}\right)+\left(k_{1}-k_{2}\right)[p]\right)},
\end{aligned}
$$

where $[p]$ is the class of a point in $C$.

Similar computations for the other component of $I_{n}(X, \beta)^{T}$, yield

$$
e\left(\mathbf{R}^{\bullet} \operatorname{pr}_{1, *}\left(\mathcal{H} \operatorname{som}(\mathcal{I}, \mathcal{I})-\mathcal{O}_{C \times X}\right)\right)^{m}=
$$

$$
\frac{\left(t_{0}-t_{1}\right)^{-k_{1}+g-1}\left(t_{0}-t_{2}\right)^{-k_{2}+g-1}}{\left(\left(t_{0}-t_{1}\right)+\left(k_{1}+2-2 g\right)[p]\right)\left(\left(t_{1}-t_{2}\right)+\left(k_{2}-k_{1}\right)[p]\right)} .
$$

Equation (4) also shows that the fixed part of

$$
\mathbf{R}^{\bullet} \operatorname{pr}_{1, *}\left(\mathcal{H o m}(\mathcal{I}, \mathcal{I})-\mathcal{O}_{C \times X}\right)
$$


is just $T_{C}$. Since we have already shown that the fixed loci are smooth, we can conclude that $\mathrm{Ob}^{f}$ is zero. Therefore, by $(3), N_{n}^{D T}(X, \beta)$ is equal to the sum of the integrals of (5) and (6) over $C$. To do the integral, we expand the fraction in terms of $[p]$ and integrate over $C$. This proves the proposition.

Remark 7. The method used here is a generalization of the method in [4]. Similar ideas also appear in [6].

\section{References}

[1] J. Bryan and R. Pandharipande, The local Promov-Witten theory of curves. Preprint math.AG/0411037, 2004.

[2] A. Gholampour, On the Gromov-Witten theory of $\mathbf{P}^{2}$-bundles over curves. Preprint math.AG/0409592, 2004.

[3] T. Graber and R. Pandharipande, Localization of virtual classes, Invent. Math. 135, no. 2, (1999) 487-518.

[4] D. Maulik, N. Nekrasov, A. Okounkov, and R. Pandharipande, Gromov-Witten theory and Donaldson-Thomas theory, I. Preprint math.AG/0312059.

[5] — Gromov-Witten theory and Donaldson-Thomas theory, II. Preprint math.AG/0406092.

[6] Y. Song, On local Donaldson-Thomas theory of curves, in preparation.

Department of Mathematics, University of British Columbia, 1984 Mathematics Road, Vancouver, B.C., Canada V6T $1 Z 2$

E-mail address: amin@math.ubc.ca

Department of Mathematics, University of British Columbia, 1984 Mathematics Road, Vancouver, B.C., Canada V6T $1 \mathrm{Z2}$

E-mail address: yinan@math.ubc.ca 